\title{
High-temperature electrical and thermal transport properties of polycrystalline $\mathrm{PdCoO}_{2}$
}

\author{
P. Yordanov $\odot,{ }^{1}$ A. S. Gibbs, ${ }^{1,2}$ P. Kaya, ${ }^{1,8}$ S. Bette, ${ }^{1}$ W. Xie $\odot,{ }^{3,4}$ X. Xiao, ${ }^{3,4}$ A. Weidenkaff, ${ }^{3,4,5}$ H. Takagi, ${ }^{1,6,7}$ and B. Keimer ${ }^{1}$ \\ ${ }^{1}$ Max-Planck Institute for Solid State Research, 70569 Stuttgart, Germany \\ ${ }^{2}$ ISIS Neutron and Muon Source, STFC Rutherford Appleton Laboratory, Chilton, Didcot, Oxon OX11 OQX, United Kingdom \\ ${ }^{3}$ Institute for Material Science, University of Stuttgart, 70569 Stuttgart, Germany \\ ${ }^{4}$ Department of Material Science, Technical University of Darmstadt, 64289 Darmstadt, Germany \\ ${ }^{5}$ Fraunhofer Research Institution for Materials Recycling and Resource Strategies (IWKS), 63457 Hanau, Germany \\ ${ }^{6}$ Institute for Functional Matter and Quantum Technologies, University of Stuttgart, 70550 Stuttgart, Germany \\ ${ }^{7}$ Department of Physics, University of Tokyo, 7-3-1 Hongo, Tokyo 113-0033, Japan \\ ${ }^{8}$ Aalen University, Materials Research Institute (IMFAA), 73430 Aalen, Germany
}

(Received 29 July 2020; accepted 6 January 2021; published 28 January 2021)

\begin{abstract}
The layered delafossite $\mathrm{PdCoO}_{2}$ has been predicted to be one of very few materials with a thermopower that is highly anisotropic and switches sign between different crystallographic directions. These properties are of interest for various applications, but have been difficult to verify because sufficiently large crystals have not been available. We report measurements of the high-temperature electrical resistivity, thermal conductivity, and thermopower of phase-pure $\mathrm{PdCoO}_{2}$ powder compacts prepared by a highly Pd-efficient synthesis route. While the electronic transport of the polycrystalline samples is dominated by that of the Pd planes, the thermopower exhibits a well-defined deviation from the in-plane character at temperatures above $600 \mathrm{~K}$, which is indicative of opposing trends in the Seebeck coefficients within and perpendicular to the delafossite layers. The experimental data are consistently described by a combination of effective-medium models based on the main axes transport quantities. The results support the predicted ambipolar thermopower anisotropy in $\mathrm{PdCoO}_{2}$.
\end{abstract}

DOI: 10.1103/PhysRevMaterials.5.015404

\section{INTRODUCTION}

$\mathrm{PdCoO}_{2}$ and $\mathrm{PtCoO}_{2}$ represent a class of layered metallic oxides that have recently stimulated a considerable amount of experimental and theoretical work due to their electronic properties and remarkable in-plane electrical conductivity, which is now known to be the highest among the oxide materials $[1,2]$. Both compounds share the delafossite crystal structure that is based on alternating triangular Pd (or Pt), and $\mathrm{CoO}_{2}$ layers (Fig. 1). The configuration of the valence electrons $\left(\mathrm{Pd}^{1+}, 4 d^{9}\right.$, or $\left.\mathrm{Pt}^{1+}, 5 d^{9}\right)$ is analogous to the one of the superconducting cuprates $\left(\mathrm{Cu}^{2+}, 3 d^{9}\right)$ [3]. The Fermi surfaces are simple and quasi-two-dimensional, giving rise to highly anisotropic transport properties [4-11]. A rapidly growing number of studies demonstrate a great potential for practical applications such as electrocatalysts [12-14], transparent electrodes [15], electron focusing devices [16], Schottky diodes [17], and laser sensing elements that rely on the large thermopower anisotropy [18,9]. Furthermore, the ambipolar nature of the thermopower, which was predicted theoretically in $\mathrm{PdCoO}_{2}$ and $\mathrm{PtCoO}_{2}$ and shown so far only

Published by the American Physical Society under the terms of the Creative Commons Attribution 4.0 International license. Further distribution of this work must maintain attribution to the author(s) and the published article's title, journal citation, and DOI. Open access publication funded by the Max Planck Society. in $\mathrm{PdCoO}_{2}$ thin films on offcut substrates [7-9], may open the way towards the fabrication of Peltier cooling elements, where no extrinsic carrier doping ( $n$ and $p$ type) and complex device architectures are necessary [19].

In view of the increased research interest in these compounds, the preparation of phase-pure powder samples for bulk applications and as targets for thin-film deposition is becoming increasingly important. Another strong motivation for our study is the need for experimental verification of the unusual Seebeck coefficient in bulk $\mathrm{PdCoO}_{2}$, as well as measurements of the thermal conductivity-a key performance characteristic for thermoelectric materials, which is very difficult to measure in thin-film samples.

In the present work, we demonstrate a highly Pd-efficient synthesis route, adapted from the metathesis reaction originally proposed by Shannon et al. [1], which allows the production of ultraclean bulk polycrystalline $\mathrm{PdCoO}_{2}$ powders. The high-temperature electrical and thermal transport properties of sintered powder pellets were measured and analyzed. For the description of the experimental data we have used the main axes transport quantities, extracted from our previous thin-film study, which were then inserted into a combination of existing analytical effective models. The procedure applied to the electrical resistivity and thermal conductivity (with electronic and phononic components) includes averaging of the anisotropic in-plane/out-of-plane responses, and evaluation of the grain boundary contribution, treated formally as a second phase and characterized by two parameters, namely the effective conductivity and porosity. 


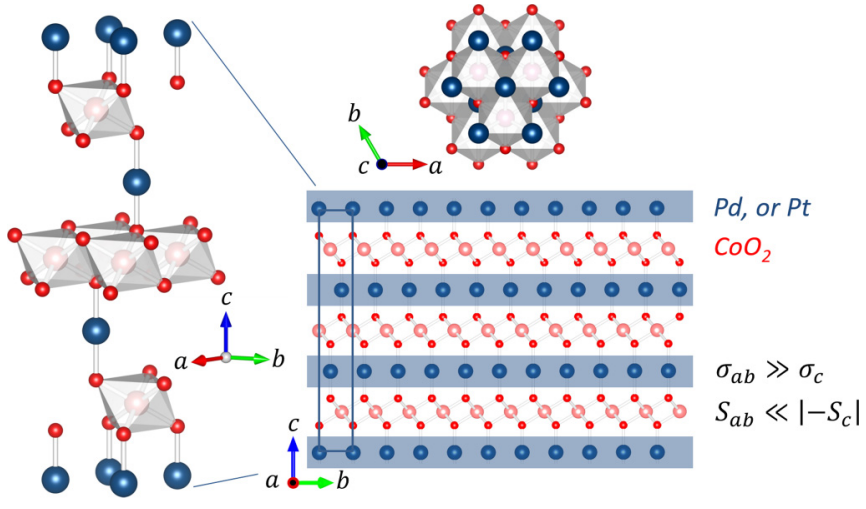

FIG. 1. Crystal structure of delafossites $\mathrm{PdCoO}_{2}$ and $\mathrm{PtCoO}_{2}$ with space group $R \overline{3} m\left(D_{3 d}^{5}\right)$ and lattice parameters $a=b=2.830 \AA$, $c=17.743 \AA$ and $a=b=2.823 \AA, c=17.815 \AA$, respectively [1] (Visualization software, VESTA 3 ). The structure consists of highly conducting $\mathrm{Pd}$, or $\mathrm{Pt}$, layers connected by $\mathrm{O}-\mathrm{Pd}(\mathrm{Pt})-\mathrm{O}$ dumbbells to insulating $\mathrm{CoO}_{2}$ (edge-shared $\mathrm{CoO}_{6}$ octahedra) blocks, both on triangular lattices. In both compounds the transport is highly anisotropic, with anisotropies of more than two orders of magnitude for the electrical conductivity $\sigma_{\mathrm{ab}} / \sigma_{c} \geqslant 100$, and as large as $\left|-S_{c}\right| / S_{\mathrm{ab}} \approx$ $|-200| / 18 \geqslant 10$ (or potentially larger in $\mathrm{PtCoO}_{2}$ ) for the Seebeck coefficient at high temperatures [7-9].

The model procedure may thus prove useful for the description of the transport properties in powder compacts comprising anisotropic grains and arbitrary porosity. Since the temperature dependence of the thermopower indicates competing positive and negative Seebeck coefficients, several conductivity-weighted average models were tested to describe the experimental data. The obtained results are in good agreement with the ambipolar thermopower anisotropy predicted in $\mathrm{PdCoO}_{2}$.

\section{SYNTHESIS OF POLYCRYSTALLINE PdCoO}

High-purity $\mathrm{LiCoO}_{2}$ was synthesized from a stoichiometric mixture of $\mathrm{Li}_{2} \mathrm{CO}_{3} 99.998 \%$ and $\mathrm{Co}_{3} \mathrm{O}_{4} 99.995 \%$, which were vacuum dried and then thoroughly ground and pelleted in an argon-filled glove box. The pellets were heated at $850{ }^{\circ} \mathrm{C}$ for $4 \mathrm{~h}$ in air and then introduced into a glove box while still hot after removal from the furnace at $200^{\circ} \mathrm{C}$. For the synthesis of $\mathrm{PdCoO}_{2}$, phase-pure $\mathrm{LiCoO}_{2}, \mathrm{Pd} 99.95 \%$, and $\mathrm{PdCl}_{2}$ 99.999\% (vacuum dried) were thoroughly mixed in an argon-filled glovebox according to the equation $\mathrm{Pd}+$ $\mathrm{PdCl}_{2}+2 \mathrm{LiCoO}_{2} \rightarrow 2 \mathrm{PdCoO}_{2}+2 \mathrm{LiCl}$. The resulting powder was pressed into pellets and sealed under vacuum in a thoroughly dried quartz ampoule with an inner quartz crucible to help withstand attack during the synthesis. The ampoule was heated at $700{ }^{\circ} \mathrm{C}$ for $80 \mathrm{~h}$ with a heating and cooling rate of $100{ }^{\circ} \mathrm{C} / \mathrm{h}$. The $\mathrm{LiCl}_{2}$ was then removed from the product by thorough washing with hot distilled water. Following this, to ensure complete removal of $\mathrm{LiCl}_{2}$, the sample was
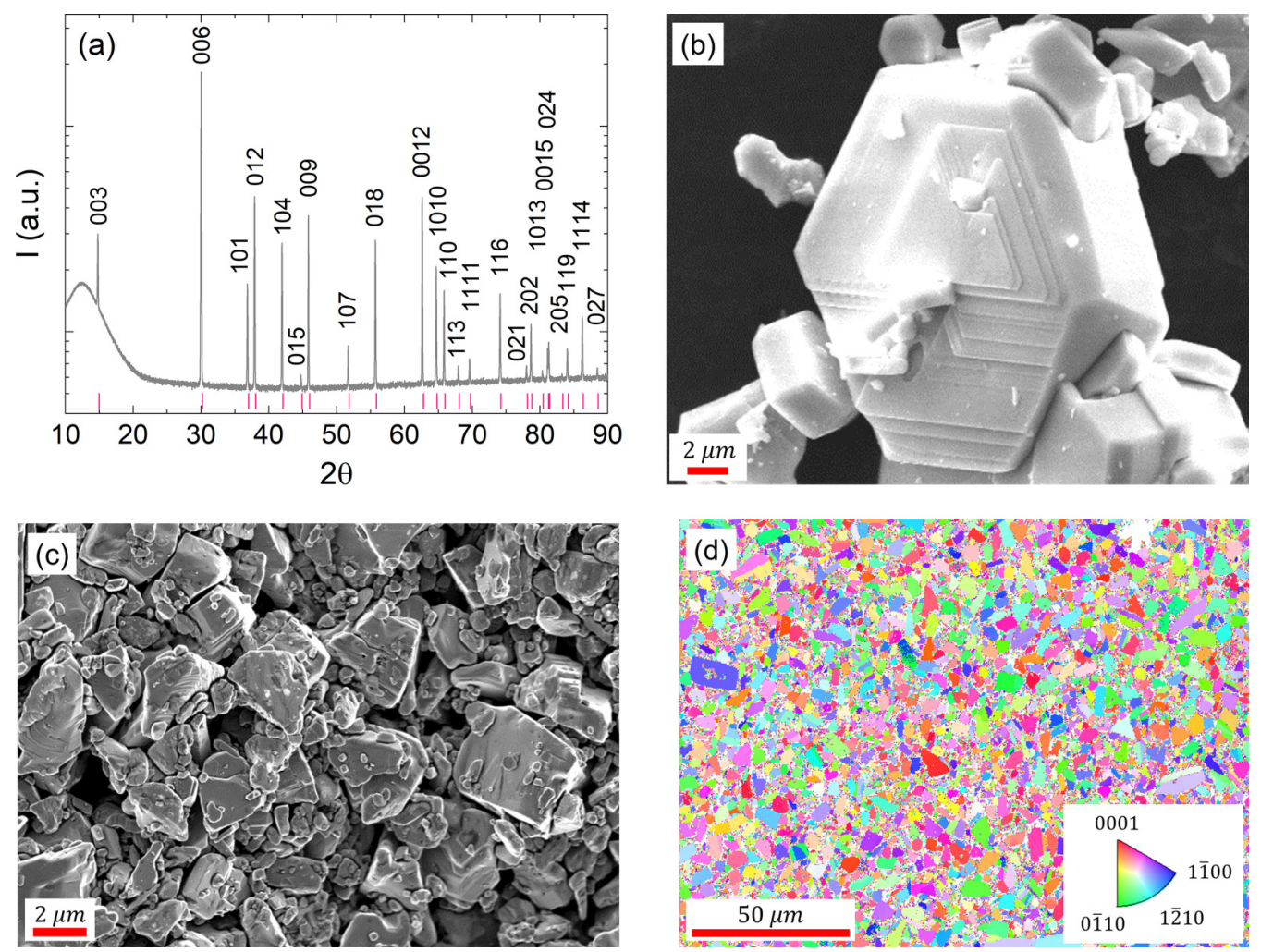

FIG. 2. (a) $\mathrm{XRD}\left(\mathrm{Cu}-K_{\alpha 1}\right.$ radiation) pattern (logarithmic scale) of as-synthesized polycrystalline $\mathrm{PdCoO}_{2}$ powder. The broad feature at low angles is due to scattering from the sample holder. The preferred orientation indicated by the higher intensity of the $00 \mathrm{l}$ reflections is due to the platelike sample morphology in Bragg-Brentano measurement geometry. The XRD pattern refinement (Pawley) yielded lattice parameters $a=b=2.8288(1) \AA, c=17.7451(5) \AA$, and $V=122.974(7) \AA^{3}$. (b), (c) SEM images of a single crystallite and sintered polycrystalline powder pellet. (d) EBSD inverse pole figure map of a sintered polycrystalline powder pellet. 
washed with dilute nitric acid before final washing with water. This process resulted in phase-pure $\mathrm{PdCoO}_{2}$ as confirmed by $\mathrm{Cu}-K_{\alpha 1}$ laboratory x-ray diffraction (XRD) [Fig. 2(a)]. Inductively coupled plasma and atomic absorption spectroscopy analysis of a representative batch yielded a $\mathrm{Pd}$ :Co ratio of $1: 1.02$, further confirming the quality of the sample.

For the characterization of polycrystalline $\mathrm{PdCoO}_{2}$, several pellets were prepared by grinding the powders in a mortar and pestle and a two-stage pressing procedure with a uniaxial and an isostatic $(800 \mathrm{kN})$ press. The pellets were then sintered in air at temperature of $\sim 750-800^{\circ} \mathrm{C}$ for $72 \mathrm{~h}$. The density of the samples was measured by the Archimedes method and found to be on average $D_{s} / D_{t} \approx 5.9 / 7.99 \approx 0.74 \pm 0.03$ of the theoretical density, with a corresponding closed porosity $\phi=1-D_{s} / D_{t} \approx 0.26 \pm 0.03$. We note that even after the sintering, the pellets were still somewhat fragile, likely due to the large powder particles with an average diameter of $d \approx 3.5 \mu \mathrm{m}$, inferred from scanning electron microscope (SEM) and electron back scatter diffraction (EBSD) maps. The inverse pole figure indicated no texture formation and therefore no preferred crystallite orientation in the sintered polycrystalline samples [Fig. 2(c) and 2(d)]. In connection to testing the polycrystalline compacts as targets for pulsed laser deposition of thin films, it is worth mentioning that we could synthesize high-quality films only by using targets consisting of large as-synthesized crystallites, and prepared under the above-described conditions. Extended (10 h, or longer) ball milling helped to decrease the particle size and enhance the compactness of the targets, but yielded films of poor quality. Spark plasma sintering did not improve the density of the compacts containing large crystallites.

\section{MEASUREMENT AND MODELING OF THE ELECTRICAL RESISTIVITY, THERMAL CONDUCTIVITY, AND SEEBECK COEFFICIENT}

The electrical resistivity $(\rho)$ and the Seebeck coefficient $(S)$ of sintered polycrystalline powder pellets were measured simultaneously in the temperature range $T=310-1000 \mathrm{~K}$ by utilizing an ULVAC (ZEM3) system equipped with $\mathrm{Pt}$ electrodes in oxygen and helium atmospheres with a pressure of 950 mbar. In the helium atmosphere, however, the sample was measurable only up to $\approx 910 \mathrm{~K}$. Above this temperature, the resistivity diverged irreversibly indicating the onset of decomposition. Each S $(T)$ data point was evaluated by three consecutive measurements with temperature difference settings of $\Delta T=20,30$, and $40 \mathrm{~K}$, respectively. The thermal conductivity $\left(\kappa=\alpha C_{p} D_{s}\right)$ was obtained from thermal diffusivity $\alpha$ and specific heat $C_{p}$ measurements in the range $T=$ $300-900 \mathrm{~K}$, in oxygen atmosphere, by a laser-flash technique system (LFA 457, Netzsch) and by a differential scanning calorimeter (DSC 404 F3 Pegasus, Netzsch), respectively.

\section{A. Electrical resistivity}

The electrical resistivity of polycrystalline $\mathrm{PdCoO}_{2}$ is presented by the open symbols in Fig. 3(a). Its behavior is dominated by that of the in-plane resistivity, where a superlinear temperature dependence $\rho_{\mathrm{ab}} \sim T^{1.41}$ was found in single crystals and thin films, over a wide temperature
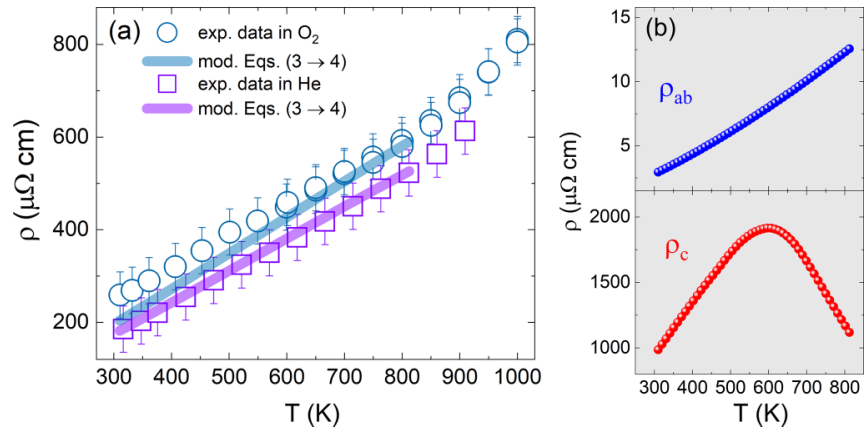

FIG. 3. (a) Experimental (open symbols) temperature-dependent resistivity $\rho$ of polycrystalline $\mathrm{PdCoO}_{2}$ for two different samples measured in oxygen (circles), and helium (squares). Solid lines show results from the model fit. (b) Main axes resistivity $\rho_{\mathrm{ab}}$ and $\rho_{c}$ of $\mathrm{PdCoO}_{2}$ used in the model description of the polycrystalline samples. Note that due to the absence of experimental data for $\rho_{\mathrm{ab}}$ and $\rho_{c}$ of single crystals at temperatures above $500 \mathrm{~K}$, data sets obtained from thin films [9] were used. $\rho_{\mathrm{ab}}$ and $\rho_{c}$ were then scaled according to the single crystal data reported in $[4,5]$. The $\rho_{\mathrm{ab}}$ and $\rho_{c}$ data extend only up to $820 \mathrm{~K}$ due to the reduced thermal stability of the thin films compared to bulk samples.

range [Fig. 3(b), upper panel[ [4,9]. The prevailing in-plane character is not surprising since $\mathrm{PdCoO}_{2}$ comprises highly conducting Pd layers separated by layers of insulating $\mathrm{CoO}_{2}$ in the perpendicular direction (Fig. 1). As a result, the $c$-axis resistivity is two orders of magnitude larger even at high temperatures. It shows a temperature dependence closer to linear, $\rho_{c} \sim T^{1.05}$, and a pronounced crossover above $\sim 600 \mathrm{~K}$ [Fig. 3(b), lower panel]. Since the investigated samples are sintered powders, the larger absolute value of the resistivity of polycrystalline $\mathrm{PdCoO}_{2}$ compared to those for single crystals and thin films along the Pd planes, where typically $\rho_{\mathrm{ab}}(300 \mathrm{~K}) \approx 2.6-12 \mu \Omega \mathrm{cm}[4,5,9]$, can be attributed to grain boundary scattering. Therefore, the model fit of $\rho$ will require a calculation of the polycrystalline conductivity $\sigma=1 / \rho$ and a quantitative description of the grain boundary contribution.

Generally, the transport in polycrystalline materials comprising randomly oriented anisotropic single crystals is a complex problem even before the influence of the grain boundaries is considered. Its description is intrinsically similar to that of two-phase composites and typically requires a numerical treatment of effective-medium theory (EMT) selfconsistent equations with specific constraints on the possible solutions [20]. Only rarely, for special cases, the results are analytical.

The most widely used bounds in terms of electrical conductivity of a polycrystal $\sigma_{\mathrm{pc}}$ are given by the arithmetic mean (upper bound, parallel resistor connection), the geometric mean (random arrangement), and the harmonic mean (lower bound, series resistor connection) of the main axes conductivities [21]; analogous bounds apply to the thermal conductivity. In $\mathrm{PdCoO}_{2}, \sigma_{a}=\sigma_{b}=\sigma_{\mathrm{ab}} \neq \sigma_{c}$ and the bounds transform to

$$
\frac{2 \sigma_{\mathrm{ab}}+\sigma_{c}}{3} \geqslant \sigma_{\mathrm{pc}} \geqslant\left(\sigma_{\mathrm{ab}}^{2} \sigma_{c}\right)^{1 / 3} \geqslant \frac{3}{\frac{2}{\sigma_{\mathrm{ab}}}+\frac{1}{\sigma_{c}}} .
$$



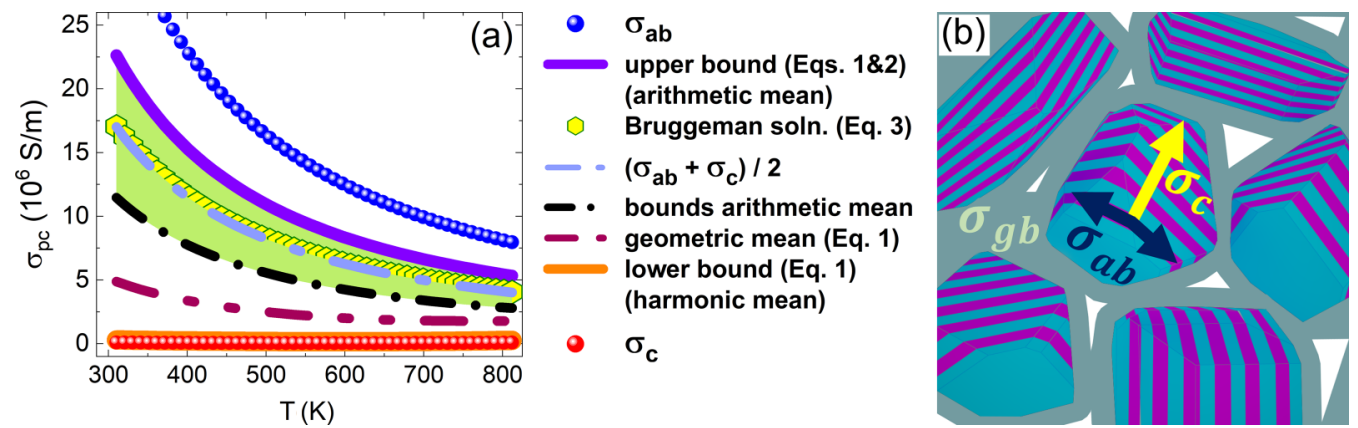

FIG. 4. (a) Bounds and solutions applicable to the electrical/thermal conductivity of polycrystalline $\mathrm{PdCoO}_{2}$. The shaded (green) area shows the range that describes best the trend in the experimental data $\sigma \rightarrow \sigma_{\mathrm{ab}} . \sigma_{\mathrm{ab}}$ and $\sigma_{c}$ correspond to $1 / \rho_{\mathrm{ab}}$ and $1 / \rho_{c}$ in Fig. 3(b). (b) Two-media model of a polycrystalline powder compact, consisting of (1) randomly oriented single crystal "cores" (defect free, fully dense material), described on the large scale by $\sigma_{\mathrm{pc}}$, and (2), a surrounding net characterized by effective grain boundary $\sigma_{\mathrm{gb}}$.

An analytical result that does not follow from the EMT and coincides with the upper bound in Eq. (1) has been suggested for the description of the electrical and thermal conductivity of anisotropic crystals with uniaxial symmetry [22,23]:

$$
\sigma_{\mathrm{pc}}=\left(2 \sigma_{\mathrm{ab}}+\sigma_{c}\right) / 3 .
$$

Next within the bounds is the Bruggeman analytical solution for the special case of uniaxial symmetry and spherical particles $[24,25]$ :

$$
\sigma_{\mathrm{pc}}=\frac{1}{4}\left[\sigma_{\mathrm{ab}}+\left(\sigma_{\mathrm{ab}}^{2}+8 \sigma_{c} \sigma_{\mathrm{ab}}\right)^{1 / 2}\right] .
$$

By inspecting the conductivity bounds and Eqs. (2) and (3) [plotted in Fig. 4(a)], we notice that the solutions positioned between the upper bound and the arithmetic mean of the upper and lower bounds (the green shaded area), can reproduce better the $\sigma \rightarrow \sigma_{\mathrm{ab}}$ behavior for $\sigma_{\mathrm{ab}} \gg \sigma_{c}$, and are therefore more suitable for the description of the experimental data. The median of this range coincides approximately with the arithmetic average of the main axes conductivity $\left(\sigma_{\mathrm{ab}}+\sigma_{c}\right) / 2$, and the Bruggeman expression [Eq. (3)].

An important further assumption is that the powder samples are statistically isotropic on the large scale. This is supported by the random spatial orientation of crystallites, as evident from Fig. 2(d). In order to describe the experimental $\rho=1 / \sigma$ data, we need to take into account the contribution of the grain boundaries. For materials with small porosity $\phi$, the effective conductivity is usually given in the form $\sigma=\sigma_{\text {fully dense }}(1-c \phi)$, where the coefficient $c$ is a fixed numerical factor $[26,27]$. The sintered polycrystalline powder pellets, however, consist of single crystals and polycrystalline aggregates of various sizes and geometries [Figs. 2(b)-2(d)] with a low degree of densification, which is a prerequisite for substantial interfacial resistance. Furthermore, the way that electrical currents flow is eventually by passing through the grain surfaces whose physical, and possibly chemical, properties may be very different compared to that of the bulk. A pertinent example is the crucial contribution of the grain surfaces in the mechanism of magnetoresistance in polycrystalline perovskite manganites [28]. As these surface modifications involve some finite depth, it is natural to assume a characteristic grain size, below which the particles can no longer be considered as objects that carry bulk properties. It is obviously difficult to introduce such a parameter precisely since this requires detailed knowledge of the depth profile characterizing the property modifications. If we assume a spherical grain (of total diameter $d_{\text {grain }}$ ) and a large contrast between the properties of the shell (with thickness $t_{\text {shell }}$ and volume $V_{\text {shell }}$ ) and the bulk core (with volume $V_{\text {core }}$ ), we can see that for $t_{\text {shell }} / d_{\text {grain }} \geqslant 1 / 10$, the volume ratio $V_{\text {shell }} / V_{\text {core }}$ is already $V_{\text {shell }} / V_{\text {core }} \gtrsim 1$, hence the particle properties will strongly deviate from that of the bulk. For shell thicknesses in order of the $\mathrm{PdCoO}_{2}$ unit cell, $t_{\text {shell }} \approx 1-2 \mathrm{~nm}$, the estimate applies to grains with diameter $d_{\text {grain }} \lesssim 10-20 \mathrm{~nm}$. It is also reasonable to assume that the onset of such a "bulk-nonbulk" crossover may occur at smaller ratios, of approximately $V_{\text {shell }} / V_{\text {core }} \approx 0.3$, corresponding to $t_{\text {shell }} / d_{\text {grain }} \approx$ $1 / 25$, and grains of diameter $d_{\text {grain }} \lesssim 25-50 \mathrm{~nm}$. In a more realistic effective model picture, the sintered low-density powder compacts can thus be viewed as a two-component system comprising randomly oriented crystallites with bulk properties embedded in a resistive environment [Fig. 4(b)]. An empirical (geometric mean-random distribution) expression, originally introduced to describe the effective thermal conductivity of two-phase systems $[29,30]$, combines the model features discussed above:

$$
\sigma=\sigma_{\mathrm{pc}}^{1-\phi} \sigma_{\mathrm{gb}}^{\phi}
$$

The two media are characterized by $\sigma_{\mathrm{pc}}$-the averaged conductivity corresponding to polycrystalline $\mathrm{PdCoO}_{2}$, and by $\sigma_{\mathrm{gb}}$ - the effective conductivity of all other components that lead to properties different from the bulk. These are defined as grain boundaries of volume fraction $\approx \phi$ and may include modified grain surfaces or shells, particles with significant $V_{\text {shell }} / V_{\text {core }}$ ratio, intergrain contacts, and voids. If $\phi$ is known, $\sigma_{\mathrm{gb}}$ can be expressed from Eq. (4), or obtained as a temperature-independent fit parameter in case of dominating defect elastic scattering contributions. The solid lines in Fig. 3(a) represent the model fits to the experimental $\rho$ data. We note that a satisfactory description could be obtained by using either Eqs. (2) and (4), or Eqs. (3) and (4). Here, only the results following from the fit by Eqs. (3) and (4) will be presented, as they provide a better description of our data. This will be demonstrated later on in the discussion of the Seebeck coefficient. For $\phi=0.26$, we obtain fit parameters $\sigma_{\mathrm{gb}} \approx 20 \mathrm{~S} / \mathrm{m}$ and $\sigma_{\mathrm{gb}} \approx 31 \mathrm{~S} / \mathrm{m}$, or $\sigma_{\mathrm{gb}}=25.5 \mathrm{~S} / \mathrm{m}$ on average, for the samples measured in oxygen and helium atmospheres, respectively. In terms of specific grain boundary 

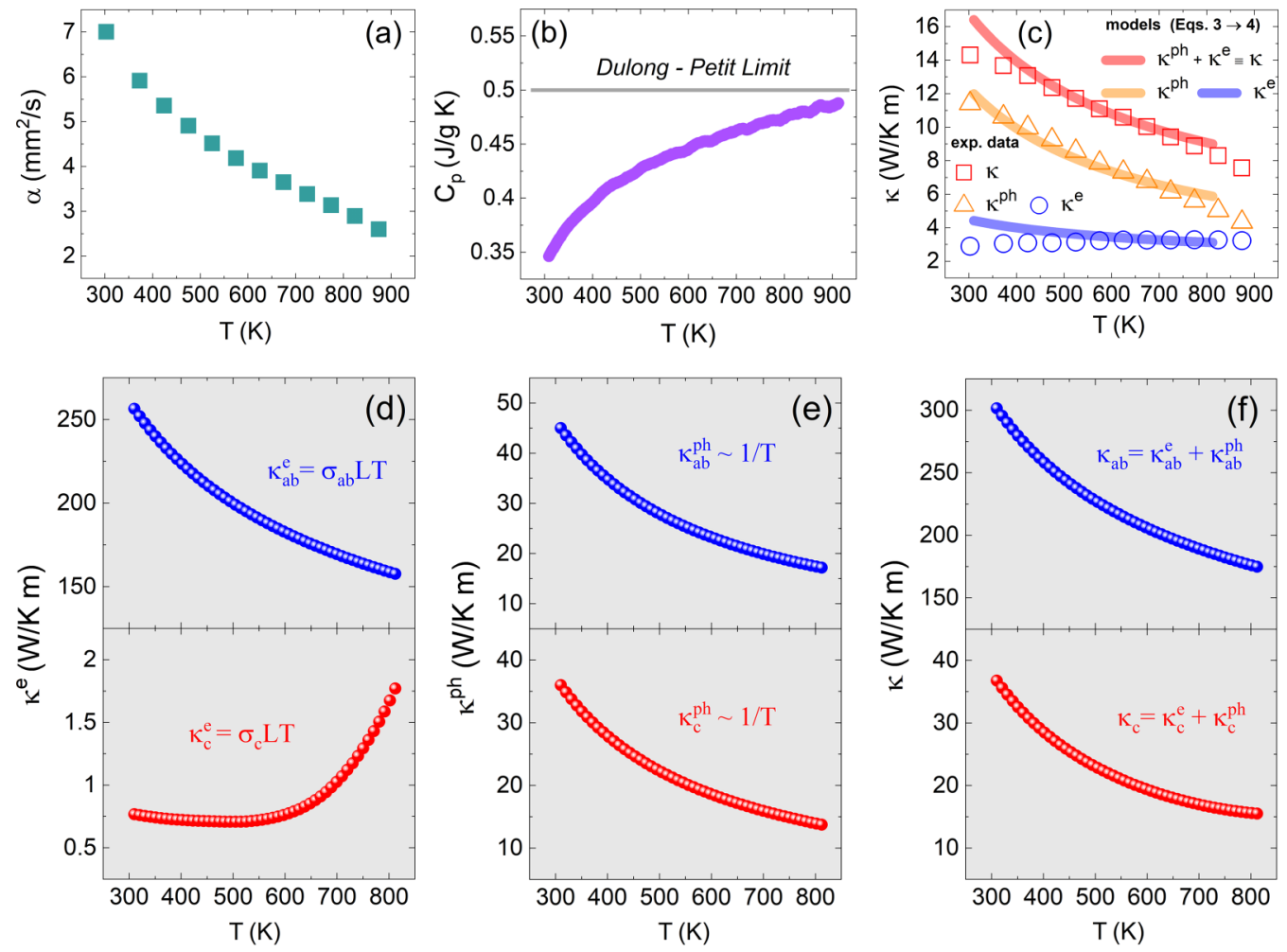

FIG. 5. (a) Thermal diffusivity $\alpha$, (b) specific heat $C_{p}$ (solid line marks the Dulong-Petit value of $C \approx 0.506 \mathrm{~J} / \mathrm{g} \mathrm{K}$ ), (c) total $\kappa$, phononic (lattice) $\kappa^{\mathrm{ph}}$, and electronic $\kappa^{e}$ thermal conductivity of polycrystalline $\mathrm{PdCoO}_{2}$. Solid lines represent model fit results. (d) Calculated main axes electronic thermal conductivity $\kappa_{\mathrm{ab}}^{e}, \kappa_{c}^{e}$, (e) simulated lattice thermal conductivity $\kappa_{\mathrm{ab}}^{\mathrm{ph}}, \kappa_{c}^{\mathrm{ph}}$, (f) main axes total thermal conductivity $\kappa_{\mathrm{ab}}, \kappa_{c}$.

resistivity,

$$
\gamma_{\mathrm{gb}}^{\mathrm{el}}=\left(1 / \sigma_{\mathrm{gb}}\right) \delta
$$

where $\delta \approx d \phi / 3$ is an estimate of the average grain boundary thickness [31,32], and $d \approx 3.5 \mu \mathrm{m}$ (the average grain diameter), we calculate $\delta \approx 300 \mathrm{~nm}$, and $\gamma_{\mathrm{gb}}^{\mathrm{el}}=1.19 \times 10^{-8} \Omega \mathrm{m}^{2}$. The obtained specific grain boundary resistivity is thus relatively large and comparable to the values reported for nanocrystalline $\mathrm{YBa}_{2} \mathrm{Cu}_{3} \mathrm{O}_{7-x}$ [33].

\section{B. Thermal conductivity}

Figures 5(a)-5(c) represent the thermal diffusivity, specific heat, and thermal conductivity of polycrystalline $\mathrm{PdCoO}_{2}$. The temperature dependence of $C_{p}$ compares quite well with the compound specific heat model data by Takatsu et al. [4], where Debye and Einstein modes were introduced to describe the contribution of the high-frequency optical phonons in $\mathrm{PdCoO}_{2}$. Generally, the thermal conductivity consists of two components: an electronic contribution $\left(\kappa^{e}\right)$, and a phononic part $\left(\kappa^{\mathrm{ph}}\right)$, which can be estimated from the difference $\kappa^{\mathrm{ph}}=$ $\kappa-\kappa^{e}$ via the Wiedemann-Franz relation $\kappa^{e}=L \sigma T$, where $L$ is the Lorenz number. Figure 5(c) (open symbols) show the experimental results for $\kappa, \kappa^{\mathrm{ph}}$, and $\kappa^{e}$ of polycrystalline $\mathrm{PdCoO}_{2}$. At room temperature, the total thermal conductivity is $\kappa \approx 15 \mathrm{~W} / \mathrm{m} \mathrm{K}$, which is much lower than the one reported for single crystals: $\kappa_{\mathrm{ab}} \approx 300 \mathrm{~W} / \mathrm{m} \mathrm{K}$ and $\kappa_{c} \approx$ $50 \mathrm{~W} / \mathrm{m} \mathrm{K}$ along the in-plane and the $c$ axis, respectively [10]. Likewise, for the lattice thermal conductivity, we obtain $\kappa^{\mathrm{ph}} \approx 12 \mathrm{~W} / \mathrm{m} \mathrm{K}$ at $300 \mathrm{~K}$, while $\kappa_{\mathrm{ab}}^{\mathrm{ph}}=45 \mathrm{~W} / \mathrm{m} \mathrm{K}$ and $\kappa_{c}^{\mathrm{ph}}=36 \mathrm{~W} / \mathrm{m} \mathrm{K}$ were theoretically calculated [11]. In the entire measured temperature range, $\kappa^{\mathrm{ph}}$ constitutes the main contribution to the total thermal conductivity $\kappa$, clearly showing that the phonons dominate the heat conduction in as-prepared polycrystalline $\mathrm{PdCoO}_{2}$. Since there are no main axes thermal conductivity data reported for $\mathrm{PdCoO}_{2}$ at temperatures above $300 \mathrm{~K}$, we can proceed with the description of the experimental results based only on estimates. These are the electronic components $\kappa_{\mathrm{ab}}^{e}, \kappa_{c}^{e}$, calculated from the $\rho_{\mathrm{ab}}$ and $\rho_{c}$, and the lattice components $\kappa_{\mathrm{ab}}^{\mathrm{ph}}, \kappa_{c}^{\mathrm{ph}}$, simulated by assuming room temperature values from [11], and an umklapp scattering $(\sim 1 / T)$ mechanism above $300 \mathrm{~K}$. The data are presented in Figs. 5(d)-5(f), along with the resulting main axes total thermal conductivity $\kappa_{\mathrm{ab}}, \kappa_{c}$.

Applying the fitting procedure separately to the electronic and the phononic components, by inserting the quantities $\kappa_{\mathrm{ab}}^{e}, \kappa_{c}^{e}$ and $\kappa_{\mathrm{ab}}^{\mathrm{ph}}, \kappa_{c}^{\mathrm{ph}}$ in Eqs. (3) and (4), and using Eq. (5), for $\phi=0.26, d=3.5 \mu \mathrm{m}$, and $\delta=300 \mathrm{~nm}$, we obtain the results represented by solid lines in Fig. 5(c) with the following fit parameters: grain boundary thermal conductivity $\kappa_{\mathrm{gb}}^{e}=$ $3 \times 10^{-4} \mathrm{~W} / \mathrm{m} \mathrm{K}$ and $\kappa_{\mathrm{gb}}^{\mathrm{ph}}=0.18 \mathrm{~W} / \mathrm{m} \mathrm{K}$, and specific thermal grain boundary resistivities $\gamma_{\mathrm{gb}}^{e}=1.01 \times 10^{-3} \mathrm{~m}^{2} \mathrm{~K} / \mathrm{W}$ and $\gamma_{\mathrm{gb}}^{\mathrm{ph}}=1.69 \times 10^{-6} \mathrm{~m}^{2} \mathrm{~K} / \mathrm{W}$. The total thermal conductivity model curve is then obtained as the sum of both fits $\kappa^{e}+\kappa^{\mathrm{ph}} \equiv \kappa$. Thus, our model results correctly reproduce the experimental observations that the grain boundaries in the as-prepared polycrystalline $\mathrm{PdCoO}_{2}$ are much less transparent to the electronic component of the thermal conductivity than to the phononic component. We note that we have tested 

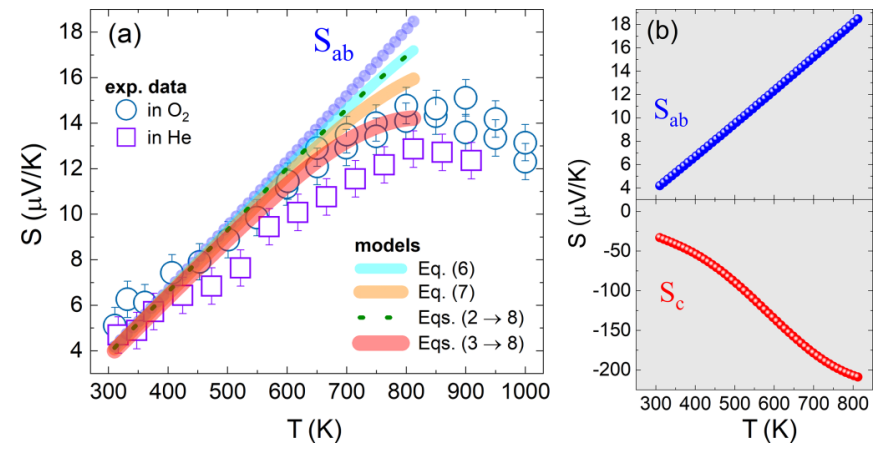

FIG. 6. (a) Experimental (open symbols) and model Seebeck coefficient (solid lines) of polycrystalline $\mathrm{PdCoO}_{2}$. The small difference in the two experimental data sets may be related to the large porosity and the different thermal contact conditions between the grains in the sample provided by the oxygen and helium atmospheres, respectively. (b) Main axes Seebeck coefficient data $S_{\mathrm{ab}}, S_{c}$ of $\mathrm{PdCoO}_{2}$, from [9].

other existing Kapitza resistance $R_{K}$-based effective models developed for nanocrystalline materials of the form $\kappa=$ $\kappa_{0} /\left(1+R_{K} \kappa_{0} / d\right)$, where $\kappa_{0}$ is the single crystal total thermal conductivity [34]. Except for a few points around room temperature and $R_{K}=2.3 \times 10^{-7} \mathrm{~m}^{2} \mathrm{~K} / \mathrm{W}$, the model results yielded too large values and do not provide a reasonable description of the experimental $\kappa$.

\section{Seebeck coefficient}

Similarly to the resistivity, the Seebeck coefficient of polycrystalline $\mathrm{PdCoO}_{2}$ (open symbols in Fig. 6) resembles that of the in-plane $S_{\mathrm{ab}}$ up to $\sim 600 \mathrm{~K}$. Only at higher temperatures, where the $\rho_{c}$ changes its character [Fig. 3(b)] and $S_{c}$ becomes large enough [Fig. 6(b)], moderate but welldefined differences between $S$ and $S_{\text {ab }}$ become visible. The temperature dependence of $S$ thus indicates a behavior governed by the conductivity-weighted average of the main axes Seebeck coefficients, in analogy to anisotropic bands systems $S=\left(\sigma_{a} S_{a}+\sigma_{b} S_{b}+\sigma_{c} S_{c}\right) /\left(\sigma_{a}+\sigma_{b}+\sigma_{c}\right)$ and systems with mixed conductivity ( $n$ - and $p$-type carriers) $S=$ $\left(\sigma_{n} S_{n}+\sigma_{p} S_{p}\right) /\left(\sigma_{n}+\sigma_{p}\right)[19,35,36]$. At low temperatures, where the $c$-axis coefficient is large $\left|-S_{c}\right|>S_{\mathrm{ab}}$, but not sufficiently larger than the in-plane $S_{\mathrm{ab}}$, and $\sigma_{\mathrm{ab}} \gg \sigma_{c}$, we observe that $S \rightarrow S_{\mathrm{ab}}$, while in the high-temperature range, the sizable negative $c$-axis thermopower $\left|-S_{c}\right| \gg S_{\mathrm{ab}}$ coincides with the nonlinear increase of $\sigma_{c}$ (downturn in $\rho_{c}$ ). It is important to note that in $\mathrm{PdCoO}_{2}$ only one band crosses the Fermi level and therefore only one carrier type is relevant for the transport. The Seebeck coefficient sign change along the different directions is thus to be attributed to the specific band structure leading to sign change in the derivative of the energy-dependent conductivity, according to the Mott formula $S \sim\{d \ln [\sigma(E)] / d E\}_{E=E_{F}}[7,8]$. Since the Seebeck coefficient does not normally require grain boundary corrections, unless conditions for energy filtering effect are fulfilled [26], we can obtain model results directly by inserting the main axes data $\rho_{\mathrm{ab}}, \rho_{c}$ and $S_{\mathrm{ab}}, S_{c}$ into the equations:

$$
S=\frac{2 \sigma_{\mathrm{ab}} S_{\mathrm{ab}}+\sigma_{c} S_{c}}{2 \sigma_{\mathrm{ab}}+\sigma_{c}}
$$

and

$$
S=\frac{\sigma_{\mathrm{ab}} S_{\mathrm{ab}}+\sigma_{c} S_{c}}{\sigma_{\mathrm{ab}}+\sigma_{c}}
$$

A reasonably good description [solid lines in Fig. 6(a)], without any fit parameters, in the entire temperature range where main axes data are available is obtained from Eq. (7). This result is somewhat surprising since due to symmetry considerations $S$ should be represented by Eq. (6), in relation to the conductivity averaging by Eq. (2). Nevertheless, Eq. (6) does not provide a satisfactory fit of the experimental data. The additional weight given to the in-plane components leads to a smaller difference between $S$ and $S_{\text {ab }}$ at high temperatures.

While some uncertainties in the main axes data $\rho_{\mathrm{ab}}, \rho_{c}$ (obtained from thin films on offcut substrates) cannot be excluded, such as a stronger downturn in $\rho_{c}$ above $T \approx 600 \mathrm{~K}$, we point out the apparent similarity of the Bruggeman solution [Eq. (3)] to the arithmetic average $\approx\left(\sigma_{\mathrm{ab}}+\sigma_{c}\right) / 2$; see Fig. 4(a). This result implies approximately equal weights of $\sigma_{\mathrm{ab}}$ and $\sigma_{c}$, which is fulfilled in Eq. (7). It is thus possible that the conductivity average following from Eq. (2), and leading eventually to Eq. (6), is not the optimal set of averaging models in our case. To crosscheck this, we will compare once again Eqs. (2) and (3) by calculating $\sigma_{\mathrm{pc}}$ and $\kappa_{\mathrm{pc}}$ and substituting them into a model for the effective Seebeck coefficient different from Eqs. (6) and (7). A suitable choice are models developed for composites comprising two different materials, such as the Halpern thermopower formula [37,20]:

$$
S=\frac{S_{\mathrm{ab}} \sigma_{\mathrm{ab}} \kappa_{c}-S_{c} \sigma_{c} \kappa_{\mathrm{ab}}}{\sigma_{\mathrm{ab}} \kappa_{c}-\sigma_{c} \kappa_{\mathrm{ab}}}+\frac{\sigma_{\mathrm{ab}} \sigma_{c} \kappa_{\mathrm{pc}}\left(S_{c}-S_{\mathrm{ab}}\right)}{\sigma_{\mathrm{pc}}\left(\sigma_{\mathrm{ab}} \kappa_{c}-\sigma_{c} \kappa_{\mathrm{ab}}\right)} .
$$

Equation (8) is a more general model that takes into account the difference in the thermal conductivity between the two phases, which is adapted here as the difference between grains of different orientations. As can be seen from Fig. 6, the use of Eqs. (2) and (8) yields a result identical to that of Eq. (6), while Eqs. (3) and (8) clearly demonstrate the difference in favor of the Bruggeman expression as the more appropriate averaging formula. The descriptions derived by Eqs. (7), and by (3) and (8), may be further improved if a precise set of high-temperature data $(T>500 \mathrm{~K})$ for $\rho_{c}$ is available. The same applies to the lattice thermal conductivity, and in particular for $\kappa_{c}^{\mathrm{ph}}$, where a nonmonotonic temperature dependence can be expected in connection to the pronounced upturn in $\kappa_{c}^{e}$ above $T \approx 600 \mathrm{~K}$. Nevertheless, the entire main axes data set [Figs. 3(b), 5(d)-5(f), and 6(b)] appears to be a good approximation of the intrinsic transport quantities of $\mathrm{PdCoO}_{2}$ in the high-temperature range. We note that if a simple extrapolation of the main axes data up to $T=1000 \mathrm{~K}$ is assumed, Eqs. (7), and (3) and (8), adequately reproduce the maximum in the thermopower due to the increasing weight of the product $\sigma_{c}\left(-S_{c}\right)$ with respect to $\sigma_{\mathrm{ab}} S_{\mathrm{ab}}$. An important factor that may need to be considered is a possible weak preferable orientation of the large as-synthesized crystallites with anisotropic shape [Fig. 2(b)] during the uniaxial press pellet formation. Consequently, even though the EBSD indicates no texture, the extremely large anisotropy in the electrical conductivity might introduce some measurable effects in the transport. These, again, will be governed by the 
conductivity-weighted average mechanism and could appear as a shift, and/or an amplitude change, of the maximum in the temperature-dependent thermopower for powder compacts of different densities (or different crystallite sizes, respectively).

\section{CONCLUSIONS}

We have demonstrated a Pd-efficient method for the synthesis of polycrystalline $\mathrm{PdCoO}_{2}$ that allows the fabrication of ultrapure material. The high-temperature electrical and thermal properties of sintered samples prepared of as-synthesized powders, with large porosity $\phi \approx 0.26$, were measured and analyzed. As can be expected, the highly conducting $\mathrm{Pd}$ layers strongly dominate the electronic transport of polycrystalline $\mathrm{PdCoO}_{2}$. It is therefore quite remarkable that the thermopower exhibits a well-defined deviation from the inplane behavior above $T \approx 600 \mathrm{~K}$, followed by a maximum, while the much more sensitive electrical resistivity remains featureless. Based on the main axes transport quantities, and a combination of existing analytical effective models, we constructed a two-step procedure, which consistently describes the experimental data. The analysis includes averaging of the main axes in-plane/out-of-plane responses, where optimal results were achieved by the Bruggeman analytical EMT solution for the uniaxial crystal symmetry, a model for the conductivities that treats the grain boundaries as randomly distributed separate phase, and a set of conductivity-weighted average formulas for the thermopower. The electrical conductivity and thermal conductivity, including its electronic and phononic components, of the powder compacts were found to be greatly suppressed compared to those in single crystals. The influence of the grain boundaries on each of these transport quantities is characterized separately by the specific grain boundary resistivity, which can be used as a basis for comparative studies. The thermopower was successfully modeled by assuming competing main axes Seebeck coefficients weighted by the respective conductivities, in agreement with the ambipolar anisotropy predicted in $\mathrm{PdCoO}_{2}[7,8]$. In polycrystalline $\mathrm{PdCoO}_{2}$, the figure of merit $Z T=S^{2} \sigma T / \kappa$, which is a measure of the conversion efficiency, remains small even at high temperatures: $Z T_{\max } \approx 5 \times 10^{-3}$. An extrapolation of our model analysis to $T=1000 \mathrm{~K}$ suggests that the $Z T=1$ mark will be difficult to reach. The only relevant case to consider would be the $c$-axis direction in single crystals, for which we estimate $Z T_{\max }^{c \text { axis }} \approx 0.8$. In contrast to energy harvesting applications, however, the strong ambipolar thermopower anisotropy in $\mathrm{PdCoO}_{2}$ may open up the way towards thin film-based devices, such as laser power sensors [18,9], and Peltier cooling elements that operate in the transverse thermoelectric regime [19].

\section{ACKNOWLEDGMENTS}

We acknowledge financial support by Deutsche Forschungsgemeinschaft (DFG, German Research Foundation) - Project No. 107745057 - CRC/TRR 80, subproject G8, and partial support by Coherent Inc., Wilsonville, OR (USA). The authors are indebted to S. Prill-Diemer, J. Nuss, M.-L. Schreiber, A. Fuchs, R. Lawitzki, G. Schmitz, L. Germann, M. Widenmeyer, C. Berger, W. Preis, W. Sitte, and the Technology Group for experimental support and discussions. We address a special acknowledgement in memory of H.-U. Habermeier for his dedication and key contributions to the research project.
[1] R. D. Shannon, D. B. Rogers, and C. T. Prewitt, Inorg. Chem. 10, 713 (1971).

[2] A. P. Mackenzie, Rep. Prog. Phys. 80, 032501 (2017).

[3] B. Keimer, S. A. Kivelson, M. R. Norman, S. Uchida, and J. Zaanen, Nature (London) 518, 179 (2015).

[4] H. Takatsu, S. Yonezawa, S. Mouri, S. Nakatsuji, K. Tanaka, and Y. Maeno, J. Phys. Soc. Jpn. 76, 104701 (2007).

[5] C. W. Hicks, A. S. Gibbs, A. P. Mackenzie, H. Takatsu, Y. Maeno, and E. A. Yelland, Phys. Rev. Lett. 109, 116401 (2012).

[6] K. P. Ong, J. Zhang, J. S. Tse, and P. Wu, Phys. Rev. B 81, 115120 (2010).

[7] K. P. Ong, D. J. Singh, and P. Wu, Phys. Rev. Lett. 104, 176601 (2010).

[8] M. E. Gruner, U. Eckern, and R. Pentcheva, Phys. Rev. B 92, 235140 (2015).

[9] P. Yordanov, W. Sigle, P. Kaya, M. E. Gruner, R. Pentcheva, B. Keimer, and H.-U. Habermeier, Phys. Rev. Mater. 3, 085403 (2019).

[10] R. Daou, R. Frésard, S. Hébert, and A. Maignan, Phys. Rev. B 91, 041113(R) (2015).

[11] L. Cheng, Q.-B. Yan, and M. Hu, Phys. Chem. Chem. Phys. 19, 21714 (2017).

[12] P.F. Carcia, R. D. Shannon, P. E. Bierstedt, and R. B. Flippen, J. Electrochem. Soc. 127, 1974 (1980).
[13] G. Li, S. Khim, C. S. Chang, C. Fu, N. Nandi, F. Li, Q. Yang, G. R. Blake, S. Parkin, G. Auffermann, Y. Sun, D. A. Muller, A. P. Mackenzie, and C. Felser, ACS Energy Lett. 4, 2185 (2019).

[14] F. Podjaski, D. Weber, S. Zhang, L. Diehl, R. Eger, V. Duppel, E. Alarcón-Lladó, G. Richter, F. Haase, A. Fontcuberta i Morral, C. Scheu, and B. V. Lotsch, Nat. Catal. 3, 55 (2020).

[15] T. Harada, K. Fujiwara, and A. Tsukazaki, APL Mater. 6, 046107 (2018).

[16] M. D. Bachmann, A. L. Sharpe, A. W. Barnard, C. Putzke, M. König, S. Khim, D. Goldhaber-Gordon, A. P. Mackenzie, and P. J. W. Moll, Nat. Commun. 10, 5081 (2019).

[17] T. Harada, S. Ito, and A. Tsukazaki, Sci. Adv. 5, 5733 (2019).

[18] H. Lengfellner, S. Zeuner, W. Prettl, and K. F. Renk, Europhys. Lett. 25, 375 (1994).

[19] C. Zhou, S. Birner, Y. Tang, K. Heinselman, and M. Grayson, Phys. Rev. Lett. 110, 227701 (2013).

[20] J. Sonntag, B. Lenoir, and P. Ziolkowski, Open J. Compos. Mater. 9, 21 (2019).

[21] K. Schulgasser, J. Phys. C: Solid State Phys. 10, 407 (1977).

[22] S. Nagata and M. Nakajima, Physica B (Amsterdam, Neth.) 92, 228 (1993).

[23] F. Yang, T. Ikeda, G. J. Snyder, and C. Dames, J. Appl. Phys. 108, 034310 (2010).

[24] D. A. G. Bruggeman, Ann. Phys. (NY) 417, 645 (1936). 
[25] S. Kumar and R. N. Singh, J. Am. Ceram. Soc. 78, 728 (1995).

[26] H. Lee, D. Vashaee, D. Z. Wang, M. S. Dresselhaus, Z. F. Ren, and G. Chen, J. Appl. Phys. 107, 094308 (2010).

[27] J. P. Angle, Z. Wang, C. Dames, and M. L. Mecatney, J. Am. Ceram. Soc. 96, 2935 (2013).

[28] S. Lee, H. Y. Hwang, B. I. Shraiman, W. D. Ratcliff, and S.-W. Cheong, Phys. Rev. Lett. 82, 4508 (1999).

[29] W. Woodside and J. H. Messmer, J. Appl. Phys. 32, 1688 (1961).

[30] D. F. C. Pribnow and J. H. Sass, J. Geophys. Res. 100, 9981 (1995).
[31] T. Mütschele and R. Kirchheim, Scr. Metall. 21, 1101 (1987).

[32] C. Suryanarayana, Bull. Mater. Sci. 17, 307 (1994).

[33] G. Wang, M. J. Raine, and D. P. Hampshire, Supercond. Sci. Technol. 30, 104001 (2017).

[34] H. C. Dong, B. Wen, and R. Melnik, Sci. Rep. 4, 7037 (2014).

[35] X. Chen, D. Parker, and D. J. Singh, Sci. Rep. 3, 3168 (2013).

[36] Y. F. Li, Y. C. Ding, B. Xiao, and Y. H. Cheng, Phys. Lett. A 380, 3748 (2016).

[37] V. Halpern, J. Phys. C: Solid State Phys. 16, L217 (1983). 